\title{
Impacto del confinamiento por COVID-19 en las plataformas de préstamo digital: el caso de GaliciaLe
}

\author{
Elena Sánchez-Muñoz* \\ *Servizo do Sistema de Bibliotecas. Xunta de Galicia \\ e-mail: elena.sanchez.munoz@xunta.gal | ORCID iD: https://orcid.org/0000-0002-4866-9999
}

Recibido: 10-10-20; 2a versión: 01-02-21; Aceptado: 22-02-21; Publicado online; 31-01-22

Cómo citar este artículo/Citation: Sánchez-Muñoz, E. (2022). Impacto del confinamiento por COVID-19 en las plataformas de préstamo digital: el caso de GaliciaLe. Revista Española de Documentación Científica, 45 (1), e317. https://doi.org/ 10.3989/ redc. 2022.1 .1843

Resumen: Las plataformas de préstamo electrónico cobraron una mayor relevancia durante el confinamiento por COVID-19, constituyéndose en una alternativa ante el cierre temporal de los servicios presenciales de las bibliotecas públicas. Se analiza el impacto de esta cuarentena en el incremento de las altas de usuarios, lectores activos y préstamos a partir de los datos generados por la plataforma de préstamo electrónico GaliciaLe, así como la repercusión de las medidas que se implantaron para facilitar el acceso de los ciudadanos a la lectura digital durante el estado de alarma. Los datos muestran un notable incremento en los indicadores analizados y permiten concluir que los servicios de préstamo digital han contribuido a suplir parcialmente a las bibliotecas públicas en la misión de promoción de la lectura que tienen encomendada estos centros. Así mismo, se discute la capacidad de las plataformas de préstamo electrónico para mantener su progresión al alza tras el confinamiento.

Palabras clave: libros electrónicos; usuarios de bibliotecas; lectura; bibliotecas públicas; estudios de uso de bibliotecas digitales; COVID-19.

\section{Impact of confinement by COVID-19 on digital lending platforms: the case of GaliciaLe}

Abstract: E-book lending platforms became more relevant during the confinement by COVID-19, reaching out to be an alternative to the temporary closure of the face-to-face services of public libraries. The impact of this quarantine on the increase in the number of users, active readers and checkouts is analyzed from the data generated by the electronic lending platforms of GaliciaLe, as well as the effects of the measures that were implemented to facilitate citizens access to digital reading during the state of alarm. The data shows a notable increase in the indicators analyzed and allow us to conclude that digital lending services have contributed to partially supply public libraries during their temporary closure in the mission of promoting reading that these centers are entrusted with. Likewise, the capacity of electronic lending platforms to maintain their upward progression after confinement is discussed.

Keywords: electronic books; library users; reading; public libraries; digital library use studies; COVID-19

Copyright: (c) 2022 CSIC. Este es un artículo de acceso abierto distribuido bajo los términos de la licencia de uso y distribución Creative Commons Reconocimiento 4.0 Internacional (CC BY 4.0). 


\section{INTRODUCCIÓN}

La puesta en marcha en 2014 de las plataformas de préstamo eBiblio, eLiburutegia y GaliciaLe inició en España las políticas de fomento de la lectura pública en torno al libro electrónico. Si bien, desde entonces, los informes estadísticos anuales reflejan una tendencia al alza en el número de préstamos y usuarios activos, estas cifras todavía están lejos de las de algunos países de nuestro entorno.

Un indicador como el número de préstamos por cada 1000 habitantes puede proporcionarnos una visión contextualizada. Si comparamos los datos procedentes de los organismos estadísticos de varios países europeos, frente a los 14,3 préstamos de libros electrónicos en España por cada 1000 habitantes durante 2018 (Ministerio de Cultura y Deporte, 2019b), Alemania alcanzó los 355,1 (Kompetenznetzwerk für Bibliotheken, 2019), Suecia los 224,4 (Sveriges Nationabibliotek, 2019) Dinamarca los 240,5 (Netbib, 2019), Finlandia los 144,4 (Opetus-ja kulttuuriministeriö, 2019) y Noruega los 115,3 (Nasjonalbiblioteket, 2019)

Por otro lado, si contraponemos los datos de préstamo en las bibliotecas públicas que ofrecía Bibliotecas Públicas españolas en cifras (Ministerio de Cultura y Deporte, 2019a) para 2018 con los de préstamo electrónico de ese mismo año, este último supuso un $2,16 \%$ sobre el total.

En el caso de Galicia, el número de préstamos por cada 1000 habitantes fue, en 2018, de 12,8 (Consellería de Cultura, Educación e Universidade, 2019), en tanto que el porcentaje de préstamos sobre el total de los efectuados en las bibliotecas públicas gallegas fue de $1,59 \%$.

Estos datos dan cuenta del escaso impacto que, hasta ahora, han tenido las plataformas de préstamo digital en España y no reflejan el potencial de este sistema (Valbuena y Cordón-García, 2019)

Debemos hacer mención a varios factores de carácter estructural, tecnológico y económico que pueden incidir en estos resultados (Sánchez-Muñoz, 2020):

1. La oferta de títulos y formatos: según el informe Panorámica de la edición española de libros 2018 (Ministerio de Cultura y Deporte, 2019c) la cifra de ISBNs inscritos en 2018 fue de 81.228. Los 18.900 ISBNs de libros electrónicos representaron el $23,2 \%$ de toda la producción. La mayoría de estos títulos se publicaron en pdf $(42,5 \%)$, seguido del ePub (33\%), Mobi $(3,1 \%)$ y otros $(21,4 \%)$. Es decir, de los 18.900 libros que se editaron en digital, 14.270 (un $75,5 \%$ ) tenían un formato con cabida en las plataformas de préstamo electrónico de las bibliotecas públicas españolas. Quedan fuera de éstas los formatos propietarios de Amazon, por lo que muchos usuarios con dispositivos Kindle no pueden descargarse los títulos que ofertan estas plataformas.

Entre las posibles causas que subyacen a la brecha existente entre los libros que se publican en papel y en digital estaría la resistencia de una parte del sector editorial, que sigue siendo reacio a publicar en formato electrónico (Alonso-Arévalo y Cordón-García, 2015), La observación directa a lo largo de 2021 y estudios como el de Giblin et al. (2019), muestran que esta renuencia empieza a ser superada por la mayoría de las editoriales que publican en formato electrónico.

Todos estos factores redundan en una menor oferta y variedad de títulos en formato digital en las bibliotecas públicas (Sánchez-Muñoz, 2019)

2. La disponibilidad de los libros: la modalidad más extendida de venta ofertada por las editoriales a las plataformas de préstamo electrónico de las bibliotecas públicas españolas es la de licencias concurrentes. Ésta consiste en la adquisición de varias licencias con un número limitado de usos (generalmente, en torno a 25), de modo que puede haber tantos lectores accediendo a una obra simultáneamente como licencias adquiridas. Así, nos hallamos ante la paradoja de que un título para el que se han comprado 5 licencias (125 usos) sólo podrá ser leído por los cinco primeros usuarios que hayan formalizado el préstamo. Los restantes lectores, aun quedando 120 usos, tendrán que hacer una reserva a la espera de que alguna de las licencias quede liberada. Este modelo no sólo es perjudicial para las bibliotecas y sus usuarios, sino que contiene la demanda y va, por tanto, en detrimento de los intereses del propio sector editorial.

3. El sistema de préstamo digital: en él confluyen las dificultades técnicas a las que se enfrentan los lectores, especialmente las relacionadas con la descarga de libros (enlazado y desenlazado de dispositivos, obtención de un ID, instalación de Adobe Digital Editions y de apps, autorización de equipos, etc.) Así mismo, la atomización del acceso a los recursos de la biblioteca implica que los lectores deban aprender a manejarse en varios entornos (el de la plataforma de préstamo digital, el del catálogo tradicional para los recursos físicos, el de otros recursos electrónicos $y$, en los casos más extremos, el de otras plataformas de préstamo electrónico). Galiciale, conformada por dos plataformas de préstamo digital (eBiblio Galicia, con obras mayoritariamente en castellano, y el Catálogo gallego 
con títulos casi exclusivamente en gallego), constituye un claro exponente de atomización de un mismo servicio para el que se requiere, además, la identificación del usuario en cada plataforma con el fin de prestar, reservar o devolver un libro. La inversión de tiempo y esfuerzo para superar la curva de aprendizaje y la resolución de incidencias de naturaleza diversa en cada entorno puede no tener, bajo el punto de vista del propio lector, un retorno suficiente. Se hace necesaria, por tanto, la implantación de tecnologías que, como las herramientas de descubrimiento (con muy escasa implantación en las bibliotecas públicas españolas), proporcionen un acceso único a todos los recursos. (Sánchez-Muñoz, 2018)

4. La resistencia al cambio: a tenor de los datos de préstamo electrónico sobre el total de préstamos, existe cierta reticencia a leer en digital entre los usuarios de las bibliotecas. Nos hallamos ante un público heterogéneo que, siguiendo la categorización de los lectores creada por Revelle y otros (2012), en función de la relación de éstos con los distintos soportes, la tecnología y su experiencia lectora, va desde los "amantes del libro" (capaces de leer en cualquier soporte, si bien asocian el placer de la lectura únicamente a lo impreso), hasta los "tecnófilos" (consideran que las ventajas del libro electrónico sobrepasan con creces cualquier desventaja), pasando por los "impresores" (eligen el libro en papel y tienen dificultades en el manejo de libros electrónicos) y los "pragmáticos" (más interesados en el contenido que en el soporte y son capaces de apreciar los pros y contras del formato digital y del papel)

Por otro lado, la irrupción del préstamo de libros electrónicos en las bibliotecas públicas ha provocado ciertas reticencias por parte de un sector minoritario de profesionales que considera este servicio como algo "ajeno" a la biblioteca y que, por tanto, no le corresponde difundir ni apoyar. Perdura una concepción de la colección como algo físico en la que no tienen cabida otros recursos externos que, unida al desconocimiento o la inseguridad ante los aspectos técnicos del préstamo digital, puede estar incidiendo en esta resistencia.

Este status quo en el que se hallaba el préstamo digital en las bibliotecas públicas españolas se vio interrumpido tras la declaración del estado de alarma por medio del Real Decreto 463/2020, de 14 de marzo, que supuso la limitación de movimientos de la ciudadanía y el cierre de las bibliotecas. En este complejo contexto, los servicios de préstamo digital de las Comunidades Autónomas irrumpieron con un crecimiento exponencial en el número de préstamos, lectores activos y nuevos usuarios.
En el caso de la Comunidad Autónoma gallega con GaliciaLe, y en respuesta a esta mayor demanda, se adoptaron las siguientes medidas:

1. Autorregistro: hasta el estado de alarma, el acceso al servicio de préstamo digital se vinculaba a que el usuario estuviera en posesión de un carné de biblioteca pública. Con las medidas de confinamiento, el Servizo do Sistema de Bibliotecas habilitó un formulario de autorregistro en ambas plataformas de GaliciaLe (eBiblio Galicia y Catálogo gallego) que permitía a cualquier ciudadano solicitar las claves de acceso. La vigencia de las mismas tras el confinamiento quedaba supeditada a que el lector solicitase el carné de una de las bibliotecas públicas de la Rede de Bibliotecas de Galicia.

2. Licencias: la apertura de las licencias en eBiblio con el fin de permitir el préstamo simultáneo de todos los usos y evitar así las reservas, ha sido una constante reivindicación del Grupo de Trabajo de Seguimiento del Servicio de Préstamo del Libro Digital, adscrito a la Comisión Técnica de Cooperación (CTC) de Bibliotecas Públicas y constituido por representantes del Ministerio de Cultura y Deporte, Comunidades Autónomas, FEMP y de bibliotecas escolares de Navarra y Extremadura.

El incremento de usuarios durante el confinamiento hizo que el número de reservas para las novedades y bestsellers se disparase y que la adquisición de más licencias por parte de las CCAA, con el fin de reforzar estos títulos, no fuese suficiente para dar respuesta a la alta demanda. Es en este contexto en el que el Grupo de Trabajo retomó la cuestión de la apertura de licencias y propuso la adopción de esta medida durante el estado de alarma. Las gestiones realizadas por el propio Ministerio de Cultura y Deporte para la subida de las novedades que acababa de adquirir, así como las de la distribuidora de contenidos digitales Libranda, posibilitó que se sumaran a esta iniciativa 32 editoriales. De este modo, los títulos que compraron tanto las CCAA como el Ministerio durante el confinamiento tuvieron sus usos abiertos al préstamo simultáneo sin limitación por número de licencias y las reservas acumuladas hasta el momento se materializaron en préstamos sin necesidad de esperas.

3. Difusión: la Consellería de Cultura, Educación e Universidade publicitó GaliciaLe y el autorregistro en los medios de comunicación gallegos con cuñas radiofónicas, entrevistas, etc. Así mismo, y bajo el hashtag \#aculturasegue, se anunció en las redes sociales tanto la noticia como un vídeo promocional. Por otro lado, las Bibliotecas Municipales de A Coruña confeccionaron varios videotuto- 
riales que fueron compartidos por otras bibliotecas en sus propias redes. Para la difusión de novedades se empleó el canal habitual para la comunicación de las mismas: el envío de correos masivos a los usuarios de GaliciaLe.

De este modo, algunos de los posibles condicionantes antes expuestos (especialmente la disponibilidad de los libros, la resistencia al cambio $y$, en cierta medida, las dificultades tecnológicas a las que se deben enfrentar los usuarios) parecieron quedar en un segundo plano a partir de la expansión de la COVID-19 y las medidas de confinamiento.

El presente trabajo tiene por objetivo analizar la repercusión que estos hechos hayan podido tener en los hábitos de lectura a través de las plataformas de préstamo.

\section{METODOLOGÍA}

Para esta investigación se ha empleado una metodología cuantitativa en la que se recogen los datos de préstamo y usuarios activos proporcionados por los módulos estadísticos de las plataformas que integran GaliciaLe (eBiblio Galicia y Catálogo gallego), así como los informes anuales (Consellería de Cultura, Educación e Universidade, 2018 y 2019)

Se ha tomado como marco de referencia temporal el periodo comprendido entre el 14/03/2020 y $31 / 05 / 2020$ y se han contextualizado los datos de préstamo y lectores activos de GaliciaLe, desde un punto de vista diacrónico, comparándolos con los de años precedentes para ese mismo periodo. También se presenta la progresión desde enero de 2020 hasta el inicio del confinamiento y se muestra la evolución de los datos desde la adopción de las dos principales medidas durante el estado de alarma: la puesta en funcionamiento del autorregistro y la apertura de los usos de las licencias adquiridas tanto por el Ministerio de Cultura y Deporte como por las Comunidades Autónomas. Así mismo, se analiza la progresión desde el final del confinamiento hasta el $31 / 10 / 2020$.

El presente estudio no avanza más allá de ese mes ya que eBiblio dejó de estar operativa en todas las CCAA desde el 26/11/2020 hasta el 9/12/2020. Esta interrupción, que no tuvo incidencia en el funcionamiento del catálogo gallego por tratarse de una plataforma propia de la Xunta de Galicia, estuvo motivada por la finalización del contrato del Ministerio de Cultura y Deporte con la empresa hasta entonces adjudicataria de la plataforma que sustentaba eBiblio (Odilo) y por los trabajos de mi- gración llevados a cabo por la ganadora de la licitación (Libranda, perteneciente al grupo canadiense De Marque).

Por otro lado, la curva de aprendizaje que requiere para el usuario el adaptarse a un nuevo entorno y a una nueva app, el elevado número de incidencias técnicas que se han registrado desde entonces, la imposibilidad de migrar la totalidad de los recursos disponibles antes de la migración y la falta de homogeneidad entre algunos datos de la nueva plataforma y los de la anterior, nos llevan a finalizar nuestro análisis en las fechas arriba indicadas.

\section{RESULTADOS}

\subsection{Préstamos}

La siguiente tabla ofrece la evolución del préstamo durante los cinco primeros meses de 2020. Se estructura en tres periodos: antes del confinamiento (hasta el $13 / 03 / 2020$ ), durante el confinamiento (desde el $14 / 03 / 2020$ hasta el $31 / 05 / 2020$ ) y tras el fin de la desescalada y del confinamiento (desde el 01/06/2020 al 31/10/2020):

Tabla I. Número de préstamos en GaliciaLe. Enero - octubre 2020

\begin{tabular}{|l|c|c|c|}
\hline & $\begin{array}{c}\text { eBiblio } \\
\text { Galicia }\end{array}$ & $\begin{array}{c}\text { Catálogo } \\
\text { gallego }\end{array}$ & Total \\
\hline Datos previos al confinamento \\
\hline Enero & 5128 & 1239 & 6367 \\
\hline Febrero & 4596 & 953 & 5549 \\
\hline Marzo (del 1 al 13) & 2502 & 503 & 3005 \\
\hline TOTAL & $\mathbf{1 2 2 2 6}$ & $\mathbf{2 6 9 5}$ & $\mathbf{1 4 9 2 1}$ \\
\hline Datos durante el confinamento \\
\hline Marzo (del 14 al 31)* & 7821 & 2748 & 10569 \\
\hline Abril ** & $18719 * * *$ & 6424 & 25962 \\
\hline Mayo & 14606 & 3629 & 18295 \\
\hline TOTAL & $\mathbf{4 1 1 4 6}$ & $\mathbf{1 2 8 0 1}$ & $\mathbf{5 4 8 2 6}$ \\
\hline Datos posteriores al confinamento \\
\hline Junio & 11605 & 1414 & 13019 \\
\hline Julio & 11001 & 878 & 11879 \\
\hline Agosto & 11506 & 853 & 12359 \\
\hline Septiembre & 9522 & 763 & 10285 \\
\hline Octubre & 9877 & 1832 & 11709 \\
\hline TOTAL & $\mathbf{5 3 5 1 1}$ & $\mathbf{5 7 4 0}$ & $\mathbf{5 9 2 5 1}$ \\
\hline
\end{tabular}

* El autorregistro se puso en funcionamiento el 20/03/2020

** Apertura de las primeras licencias adquiridas bajo la modalidad de usos concurrentes.

$* * *$ Se incluyen las descargas de audiolibros accesibles sin préstamo previo. 
Debemos hacer mención a los siguientes elementos que han convergido a lo largo del periodo que va desde el 14/03/2020 hasta el 31/05/2020:

- El inicio del confinamiento el 14/03/2020.

- La puesta en funcionamiento del autorregistro de GaliciaLe el 20/03/2020.

- La apertura de las licencias adquiridas para eBiblio Galicia durante el confinamiento a partir del 15/04/2020.

Por otro lado, entre el $01 / 06 / 2020$ y el $31 / 10 / 2020$ es necesario destacar:

- La supresión del formulario de autorregistro el $21 / 06 / 2020$.

- El borrado, el 26/09/2020, de los lectores que no hubieran solicitado el carné de una de las bibliotecas públicas de la Red.

La comparativa entre el número de préstamos realizados desde el 01/01/2020 hasta el $13 / 03 / 2020$ (73 días) con los préstamos efectuados desde el 14/03/2020 hasta el 31/05/2020 (79 días), muestra cómo para un periodo de similar duración, el total de préstamos ha aumentado un $326 \%$ en el caso de eBiblio Galicia y un $374 \%$ en el del Catálogo gallego.

Se observa un descenso moderado en el número préstamos entre enero y febrero, quizá motivado por el fin del efecto de las novedades editoriales de Navidad. Es entre febrero y marzo cuando se produce un crecimiento intermensual más acusado $(124,6 \%$ en eBiblio Galicia y $241,1 \%$ en el catálogo gallego), en tanto que de abril a mayo, coincidiendo con el inicio de la desescalada, las cifras descienden drásticamente $(-22 \%$ en eBiblio Galicia y $-43,5 \%$ en el catálogo gallego).

De junio a octubre se aprecia una disminución en el número de préstamos, especialmente significativa entre mayo y junio $(-20,54 \%$ en eBiblio Galicia y $-61 \%$ en el catálogo gallego), así como entre agosto y septiembre $(-17,24 \%$ en eBiblio Galicia y $-10,55 \%$ en el catálogo gallego)

La Figura 1 permite contextualizar los datos de préstamo del período comprendido entre enero y mayo de 2020 con los de esos mismos meses en años precedentes, en tanto que la Figura $2 \mathrm{com}-$ para los datos de junio a octubre de 2020 con los de los años anteriores.

En ambos gráficos se observa un patrón similar, si bien se presentan diferencias entre las dos plataformas. El catálogo gallego mantiene un nivel de crecimiento sostenido desde 2015 y muestra un incremento abrupto entre enero y mayo de
Figura 1. Evolución anual del número de préstamos en GaliciaLe. Enero-mayo.

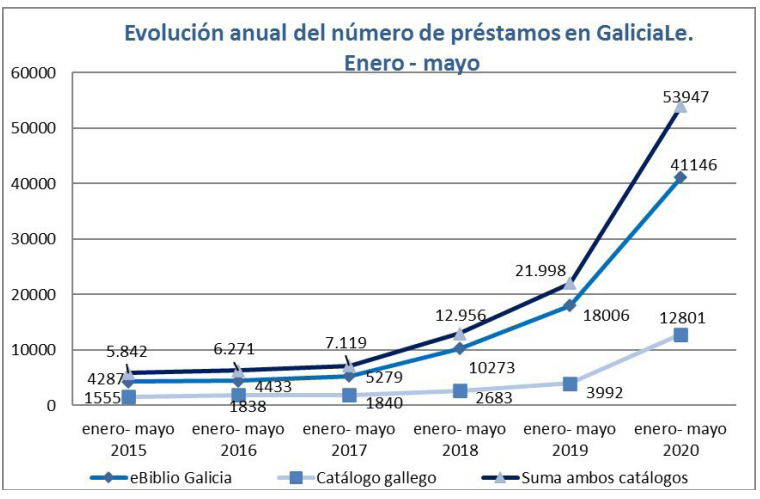

Figura 2. Evolución anual del número de préstamos en GaliciaLe. Junio-octubre.

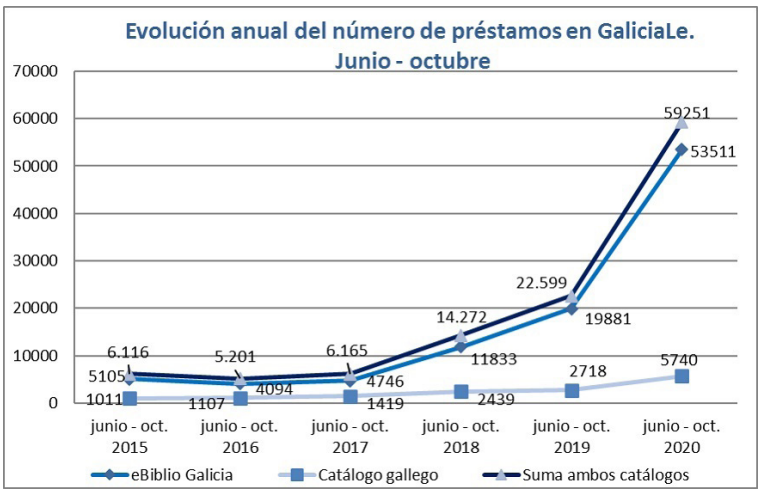

2020 (de un $220,7 \%$ respecto a ese mismo período en 2019). Entre junio y octubre de 2020 el aumento es de un $111,2 \%$ respecto a junio/octubre de 2019.

eBiblio Galicia, sin embargo, inicia una curva ascendente en 2018 , con un incremento del 169,2\% entre junio y octubre de 2020 respecto a esos mismos meses de 2019, superando el crecimiento que se produjo entre enero/mayo de 2020 respecto a enero/mayo de 2019 (un 128,5\%)

Los datos, en términos de número de préstamos anuales de cada uno de los catálogos que constituyen GaliciaLe en el período comprendido entre el 20 de octubre de 2014 (fecha de inicio del servicio) y el 31 de mayo de 2020, aparecen reflejados en la tabla II.

La comparación entre los préstamos de los primeros meses de 2020 (127364), con los habidos entre el $20 / 10 / 2014$ y el $31 / 12 / 2019$ (138484) permite ver la dimensión del crecimiento exponencial de GaliciaLe en este último año (Tabla II). 
Tabla II. Evolución anual del número de préstamos. Octubre 2014 - mayo 2020.

\begin{tabular}{|c|c|c|c|}
\hline & eBiblio Galicia* & Catálogo gallego & TOTAL \\
\hline $2014(20 / 10 / 2014-31 / 12 / 2014)$ & 2332 & 1014 & 3346 \\
\hline 2015 & 10204 & 3014 & 13218 \\
\hline 2016 & 10651 & 3476 & 14127 \\
\hline 2017 & 12928 & 4009 & 16937 \\
\hline 2018 & 28240 & 6472 & 34712 \\
\hline 2019 & 47519 & 8625 & 56144 \\
\hline $2020(01 / 01 / 2020-31 / 10 / 2020)$ & 105343 & 22021 & 127364 \\
\hline
\end{tabular}

*Se incluyen las descargas de audiolibros accesibles sin préstamo previo.

\subsection{Usuarios activos}

La Tabla III muestra la evolución de los usuarios activos (entendidos como aquéllos que hicieron, en el periodo de referencia, al menos un préstamo en alguno de los dos catálogos que constituyen GaliciaLe). Los datos de cada catálogo se presentan de manera desagregada (no se ofrece un total dado que una parte de los lectores son usuarios activos de ambos).

Tabla III. Número de usuarios activos en GaliciaLe. Enero - octubre 2020.

\begin{tabular}{|l|c|c|}
\hline \multicolumn{2}{|c|}{$\begin{array}{c}\text { eBiblio } \\
\text { Galicia }\end{array}$} & $\begin{array}{c}\text { Catálogo } \\
\text { gallego }\end{array}$ \\
\hline \multicolumn{2}{|l|}{ Datos previos al confinamiento } \\
\hline Enero & 1876 & 664 \\
\hline Febrero & 1711 & 528 \\
\hline Marzo (del 1 al 13) & 1427 & 282 \\
\hline \multicolumn{2}{|l|}{ Datos durante el confinamiento } \\
\hline Marzo (del 14 al 31) * & 3492 & 1379 \\
\hline Abril ** & 5755 & 3272 \\
\hline Mayo & 4440 & 1755 \\
\hline Datos posteriores al confinamiento \\
\hline Junio & 3637 & 695 \\
\hline Julio & 3347 & 394 \\
\hline Agosto & 3473 & 376 \\
\hline Septiembre & 3215 & 372 \\
\hline Octubre & 3428 & 1058 \\
\hline
\end{tabular}

* El autorregistro se puso en funcionamiento el 20/03/2020 ** Apertura de las primeras licencias adquiridas bajo la modalidad de usos concurrentes.

Nuevamente, se aprecia cómo el inicio del confinamiento $y$, muy especialmente, la puesta en funcionamiento del servicio de autorregistro en abril, supusieron un notable incremento en el número de usuarios activos.
Si bien el mayor descenso se produjo, igualmente, entre mayo y junio $(-18,08 \%$ en eBiblio Galicia y $-60,39 \%$ en el catálogo gallego), en los meses posteriores, la cifras de eBiblio Galicia se han mantenido estables, a diferencia del catálogo gallego, que sufrió otra fuerte disminución entre junio y julio $(-43 \%)$ y volvió a recuperarse de septiembre a octubre, con un crecimiento intermensual del $184,4 \%$, quizá motivado por el inicio del curso escolar y la prescripción de lecturas obligatorias en los centros educativos.

Las Figura 3 y 4 permiten volver a contextualizar los datos, en este caso de usuarios activos, en los períodos de enero a mayo y de junio a octubre, dentro de las series anuales desde 2015 a 2020.

En ambos gráficos se observan patrones más diferenciados. En el caso de eBiblio Galicia, la figura 3 muestra cómo la tendencia al alza más significativa se inicia en 2018 con un $55,7 \%$ respecto a 2017, continúa en 2019 con un 63,3\% respecto a 2018 , y se triplica en 2020 con un $188,8 \%$. Hasta 2019, el catálogo gallego presenta una evolución lineal con crecimientos sostenidos. Es en ese año cuando se produce un aumento significativo (del $58,6 \%$ respecto a 2018), que se quintuplica en 2020 con un $301 \%$.

En la figura 4, sin embargo, el crecimiento se inicia, para ambos catálogos, en 2018, con un aumento en eBiblio Galicia del $92,6 \%$ y del $57,9 \%$ en el catálogo gallego. En 2019, el crecimiento de eBiblio está en el $60,6 \%$ respecto a 2018 y el del catálogo gallego en tan sólo un $12,3 \%$. Será entre 2019 y 2020 cuando podamos hablar de un crecimiento más igualado entre ambos catálogos (132,6\% en eBiblio Galicia y $107,9 \%$ en el catálogo gallego)

Los datos, en términos de número de usuarios activos de cada uno de los catálogos que constituyen GaliciaLe entre el 20 de octubre de 2014 (fecha de inicio del servicio) y el 31 de octubre de 2020, aparecen reflejados en la tabla IV. 
Figura 3. Evolución anual del número de usuarios activos en GaliciaLe. Enero-mayo.

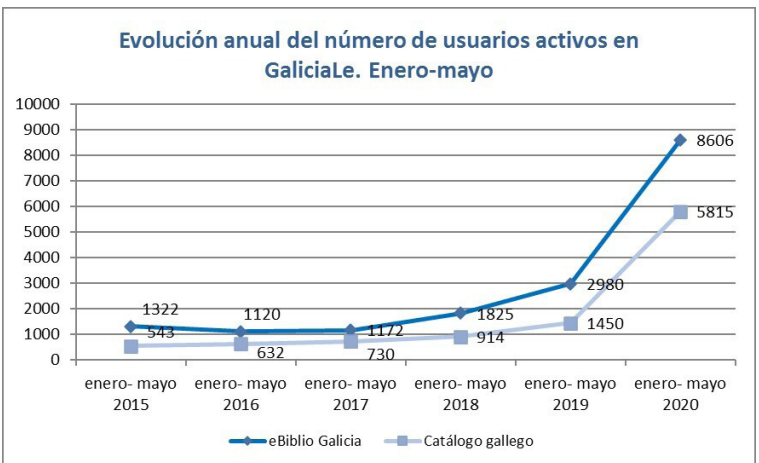

Figura 4. Evolución anual del número de usuarios activos en GaliciaLe. Junio-octubre.

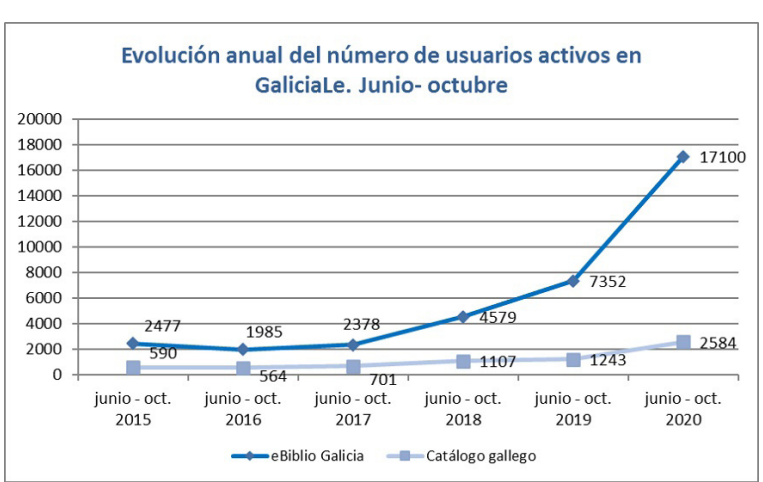

Tabla IV. Evolución anual del número de usuarios activos. Octubre 2014 - mayo 2020.

\begin{tabular}{|c|c|c|}
\hline & $\begin{array}{l}\text { eBiblio } \\
\text { Galicia }\end{array}$ & $\begin{array}{l}\text { Catálogo } \\
\text { gallego }\end{array}$ \\
\hline $\begin{array}{l}2014 \\
(20 / 10 / 2014-31 / 12 / 2014)\end{array}$ & 985 & 427 \\
\hline 2015 & 2148 & 969 \\
\hline 2016 & 1438 & 1051 \\
\hline 2017 & 2012 & 1224 \\
\hline 2018 & 3354 & 1787 \\
\hline 2019 & 4902 & 2398 \\
\hline $\begin{array}{l}2020 \\
(01 / 01 / 2020-31 / 10 / 2020)\end{array}$ & 8606 & 5815 \\
\hline
\end{tabular}

El número de usuarios activos en los diez primeros meses de 2020 arroja cifras muy superiores a las de los años precedentes $y$, al igual que en el caso de los préstamos, son un reflejo del despegue de GaliciaLe en 2020.

\subsection{Autorregistro y apertura de licencias}

La posibilidad de que cualquier ciudadano se autorregistrase para acceder al préstamo electrónico sin necesidad de estar en posesión de un carné de bi- blioteca pública, así como la apertura de las licencias para la lectura simultánea, han sido dos elementos novedosos en Galiciale y constituyeron las principales medidas adoptadas durante el estado de alarma.

\subsubsection{Autorregistro}

El servicio de autorregistro en GaliciaLe, desde cualquiera de las dos plataformas que lo conforman (eBiblio Galicia y el Catálogo gallego), se habilitó el 20/03/2020.

La condición para el mantenimiento de esta prestación tras el confinamiento era que el usuario se acercase a una biblioteca pública para solicitar que se le expidiera el carné.

Desde la Consellería de Cultura, Educación e Universidade se habilitó un formulario para tramitar el alta por medio del autorregistro en GaliciaLe adaptando el ya existente para solicitar el carné en las bibliotecas con el programa de gestión Koha. Esta adaptación permitió que los ciudadanos residentes en localidades cuya biblioteca pública empleara otro sistema de gestión bibliotecaria, también pudieran autorregistrarse.

La Tabla V muestra la evolución en el número de usuarios autorregistrados en 2020, antes de habilitar el formulario en GaliciaLe (del 1/01/2020 al $19 / 03 / 2020$ ), durante el tiempo en que estuvo activado (del 20/03/2020 al 21/06/2020) y después de deshabilitarlo (del 22/06/2020 al 31/10/2020):

Tabla V. Número de usuarios autorregistrados. Enero - octubre 2020

\begin{tabular}{|l|c|}
\hline \multicolumn{2}{|l|}{$\begin{array}{l}\text { Datos previos a la puesta en funcionamiento del } \\
\text { autorregistro en GaliciaLe }\end{array}$} \\
\hline Enero & 320 \\
\hline Febrero & 289 \\
\hline Marzo (del 1 al 19) & 136 \\
\hline \begin{tabular}{l}
$|l|$ \\
Datos durante la puesta en funcionamiento del \\
\hline Marzo (del 20 al 31)
\end{tabular} & 2021 \\
\hline Abril & 3235 \\
\hline Mayo & 665 \\
\hline Junio (del 1 al 21) & 204 \\
\hline $\begin{array}{l}\text { Datos desde la supresión del autorregistro en } \\
\text { GaliciaLe }\end{array}$ & 65 \\
\hline Junio (del 22 al 30) & 432 \\
\hline Julio & 206 \\
\hline Agosto & 390 \\
\hline Septiembre & 493 \\
\hline Octubre
\end{tabular}


Desde el 20/03/2020 al 31/05/2020 (73 días), el número de usuarios autorregistrados multiplicó por ocho la cifra de lectores autorregistrados exclusivamente para solicitar el carné de biblioteca desde el 01/01/2020 al 19/03/2020 (79 días). El mayor incremento se produjo en abril, pero los autorregistros descendieron considerablemente en mayo y, muy especialmente en junio y agosto, manteniéndose en cifras similares a las de cualquier mes anterior a la implantación del formulario en GaliciaLe.

La supresión del servicio de autorregistro en junio, así como el borrado de usuarios autorregistrados que no hubieran acudido a una biblioteca pública para formalizar su carné, no parece haber tenido la incidencia que cabía esperar a priori, a tenor de los datos obtenidos acerca de los usuarios que permanecieron activos entre junio y octubre (Véanse Tabla III y Figura 4)

De este modo, de los 5561 lectores que se autorregistraron durante el confinamiento, se suprimieron 4519 registros en septiembre (un $81,17 \%$ ), correspondientes a usuarios que no solicitaron el carné o que estaban duplicados y ya existían en el módulo de Koha antes del estado de alarma. A pesar de esto, no sólo no se aprecia un descenso en los usuarios activos entre septiembre y octubre, sino que se ha producido un incremento notable, del $184,4 \%$ arriba apuntado, en el Catálogo gallego.

Así mismo, el aumento de préstamos en octubre respecto a febrero, mes anterior a la declaración del estado de alarma, ha alcanzado un $111 \%$ (de 5559 a 11709), en tanto que el de usuarios activos ha sido de un $100,3 \%$ en ambos catálogos.

\subsubsection{Apertura de licencias}

Debemos destacar que el modelo de adquisición del Catálogo gallego, que tiene su origen en los acuerdos alcanzados entre la Xunta de Galicia y los editores gallegos, permite el préstamo simultáneo de todos los usos adquiridos. Esto evita, en consecuencia, que los usuarios tengan que hacer reservas y da acceso inmediato a la lectura de cualquier libro. Así mismo, ha propiciado que el catálogo esté preparado para una situación de excepcionalidad como la acaecida.

Por contra, la modalidad de adquisición vigente hasta el confinamiento y tras él para todas las Comunidades Autónomas que cuentan con eBiblio es el límite de uso simultáneo en las licencias. Este modelo causa un alto número de reservas, largos tiempos de espera e, incluso, provoca que haya reservas que no llegan a realizarse (cuando un libro alcanza los tres meses de espera, el sistema no permite que el usuario haga una reserva). Estos elementos hicieron que eBiblio no estuviera preparada para una eventualidad como la del confinamiento, con una alta demanda de préstamo electrónico.

El fuerte incremento de los préstamos durante la cuarentena estaba ejerciendo una gran presión sobre la colección, que sólo podía reducirse comprando licencias bajo un nuevo modelo. Con el fin de proporcionar una solución a esta situación, se llegó a un acuerdo con 32 editores y con Libranda, uno de los principales distribuidores de contenidos digitales a nivel nacional, para que los títulos adquiridos hasta el final del confinamiento, tanto por las Comunidades Autónomas como por el Ministerio de Cultura y Deporte, tuvieran licencias abiertas para

Figura 5. Evolución de los préstamos y de las reservas hechos efectivos diariamente. Enero-mayo.

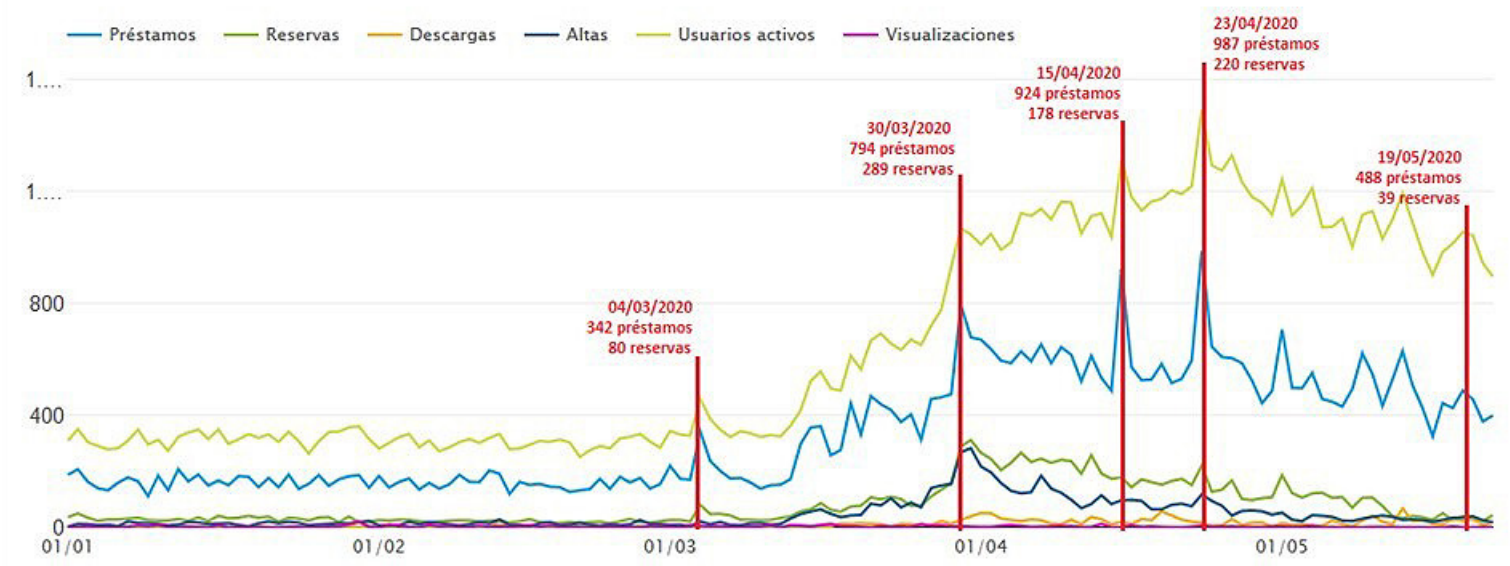

Fuente: eBiblio Galicia 
Tabla VI. Ratio reservas/préstamos en eBiblio Galicia. Junio 2019 - octubre 2020

\begin{tabular}{|c|c|c|c|}
\hline & Reservas* & Préstamos* & $\begin{array}{c}\text { Ratio préstamos/ } \\
\text { reservas }\end{array}$ \\
\hline Junio 2019 & 697 & 3562 & 0,19 \\
\hline Julio 2019 & 669 & 3739 & 0,17 \\
\hline Agosto 2019 & 695 & 4256 & 0,16 \\
\hline Septiembre 2019 & 600 & 3802 & 0,15 \\
\hline Octubre 2019 & 854 & 4543 & 0,18 \\
\hline Noviembre 2019 & 929 & 4403 & 0,21 \\
\hline Diciembre 2019 & 909 & 4909 & 0,18 \\
\hline Enero 2020 & 943 & 5099 & 0,18 \\
\hline Febrero 2020 & 620 & 4536 & 0,13 \\
\hline Marzo 2020 & 2513 & 10071 & 0,24 \\
\hline Abril 2020 & 5559 & 18017 & 0,30 \\
\hline Mayo 2020 & 1835 & 14109 & 0,13 \\
\hline Junio 2020 & 468 & 11605 & 0,04 \\
\hline Julio 2020 & 391 & 11001 & 0,03 \\
\hline Agosto 2020 & 1470 & 11506 & 0,12 \\
\hline Septiembre 2020 & 862 & 9522 & 0,09 \\
\hline Octubre 2020 & 825 & 9877 & 0,08 \\
\hline
\end{tabular}

el préstamo simultáneo y concurrente de todos sus usos. Este acuerdo permitió que los lectores pudieran acceder a la lectura de un libro de manera inmediata, y evitó la acumulación de reservas y las esperas de días e, incluso, semanas.

Este nuevo modelo de adquisición se aplicó progresivamente en eBiblio Galicia desde el $15 / 04 / 2020$, fecha en la que se cargaron las primeras licencias abiertas. La figura 5 muestra la evolución de los préstamos y de las reservas hechos efectivos diariamente desde el 01/01/2020 hasta el 22/05/2020. Dentro de ese periodo, hemos tomado cinco fechas de referencia en las que se produjo un mayor pico en el número de préstamos:

Diez días antes de la declaración del estado de alarma se incorporaron novedades con el consiguiente repunte de préstamos y de reservas y una ratio de reservas hechas efectivas/préstamos hechos efectivos de 0,23.

El 30/03/2020, en pleno confinamiento y con el servicio de autorregistro habilitado 10 días antes, la ratio ascendió a 0,36.

El 15/04/2020, fecha en la que se reforzaron 83 títulos ya existentes en eBiblio Galicia con las primeras licencias accesibles bajo esta nueva modalidad, la ratio disminuyó a 0,19 . Debemos tener en cuenta que el 14/04/2020 se produjeron 488 prés- tamos. Es decir, la incorporación de esas licencias con usos concurrentes propició que el 15/04/2020 se materializaran 436 préstamos más, que de otra manera, hubieran seguido en espera en forma de reservas.

El 23/04/2020 se incorporaron al catálogo licencias con usos concurrentes para reforzar títulos ya existentes en el catálogo, así como novedades. Se observa otro fuerte ascenso en el número de préstamos respecto al día anterior y se supera el récord de préstamos previo. La ratio de reservas, sin embargo, siguió en un nivel moderado con 0,22.

A medida que las fases de desconfinamiento fueron avanzando, tanto el número de préstamos como el de reservas descendió, pero la ratio reservas/préstamos siguió una progresión descendente, alcanzando su nivel más bajo $(0,07)$ el 19/05/2020, fecha en la que también se produjo una nueva incorporación de títulos y licencias abiertas.

La tabla VI presenta la evolución de la ratio reservas hechas efectivas mensualmente/préstamos hechos efectivos mensualmente desde junio de 2019 hasta octubre de 2020.

Desde julio de 2019 hasta enero de 2020 se observan variaciones intermensuales en la ratio reservas/préstamos que van desde el $-16,6 \%$ de noviembre a diciembre (de 0,21 a 0,18 ) al $20 \%$ de septiembre a octubre (de 0,15 a 0,18 ). La ma- 
yor variabilidad se presenta entre enero y febrero, con un fuerte descenso en la ratio (de $-27,7 \%$ ), que podría estar motivado por el fin del efecto de las novedades editoriales de Navidad: lectores que quieren tomar en préstamo los bestsellers en cuanto se publican y, en caso de no estar disponibles, hacen una reserva. Para el mes de febrero, una buena parte de las reservas hechas entre noviembre y diciembre ya se han podido materializar en préstamos que, una vez devueltos, han dejado licencias libres.

El mayor incremento (un $84,6 \%$ ) se produce entre febrero y marzo, con la convergencia en marzo de varios elementos: la incorporación de novedades el día 4, el inicio del confinamiento el 14, y la puesta en funcionamiento del autorregistro el 20.

Entre marzo y abril el aumento de la ratio es de un $25 \%$ (de 0,24 a 0,30). El refuerzo de 83 títulos con licencias abiertas el 15/04/2020, así como la posterior incorporación de más licencias y nuevos títulos bajo la misma modalidad el 23/04/2020, puede explicar un incremento más moderado de la ratio reservas/préstamos a pesar del aumento del $78,9 \%$ en los préstamos y del $107,2 \%$ en los usuarios activos.

No obstante, son los datos de mayo los que reflejan el impacto de la creciente incorporación de licencias abiertas entre este mes y el anterior. La ratio alcanza sus niveles más bajos, sólo por de- trás de febrero, a pesar de que la actividad ha sido mucho mayor en mayo (los préstamos y las reservas casi triplican las cifras de febrero). De haberse producido la adquisición de novedades de mayo en las mismas condiciones anteriores al confinamiento, pero con el nivel de demanda de ese momento, la ratio hubiera seguido una tendencia al alza en la que se habrían materializado más reservas y menos préstamos.

El hecho de que las novedades adquiridas durante el confinamiento conserven el préstamo concurrente de usos ha seguido teniendo efectos muy positivos en la ratio reservas/préstamos durante junio $(0,04)$ y julio $(0,03)$. En agosto, sin embargo, con la incorporación de novedades el 31/07/2020 bajo las condiciones anteriores al confinamiento, se aprecia un notable incremento en esta ratio $(0,12)$, que, con una ligera baja, se mantiene estable en septiembre $(0,09)$ y octubre $(0,08)$

Si comparamos la ratio de julio de 2020 (la más baja de toda la serie), con la de julio de 2019, el descenso es de un $-82,3 \%$.

Otro indicador de interés para medir el impacto de la apertura de las licencias adquiridas durante el confinamiento es la ratio reservas/usuarios que se presenta en la tabla VII:

Al igual que para la tasa reservas/préstamos, entre junio de 2019 y febrero de 2020, la ratio

Tabla VII. Ratio reservas/usuarios activos en eBiblio Galicia. Junio 2019 - octubre 2020

\begin{tabular}{|l|c|c|c|}
\hline & Reservas & Usuarios activos & $\begin{array}{c}\text { Ratio usuarios } \\
\text { activos/reservas }\end{array}$ \\
\hline Junio 2019 & 697 & 1315 & 0,53 \\
\hline Julio 2019 & 669 & 1402 & 0,47 \\
\hline Agosto 2019 & 695 & 1502 & 0,46 \\
\hline Septiembre 2019 & 600 & 1469 & 0,40 \\
\hline Octubre 2019 & 854 & 1664 & 0,51 \\
\hline Noviembre 2019 & 929 & 1666 & 0,55 \\
\hline Diciembre 2019 & 909 & 1782 & 0,51 \\
\hline Enero 2020 & 943 & 1876 & 0,50 \\
\hline Febrero 2020 & 620 & 1771 & 0,35 \\
\hline Marzo 2020 & 2513 & 3840 & 0,65 \\
\hline Abril 2020 & 5559 & 5755 & 0,96 \\
\hline Mayo 2020 & 1835 & 4440 & 0,41 \\
\hline Junio 2020 & 468 & 3637 & 0,12 \\
\hline Julio 2020 & 391 & 3347 & 0,11 \\
\hline Agosto 2020 & 1470 & 3473 & 0,42 \\
\hline Septiembre 2020 & 862 & 3215 & 0,26 \\
\hline Octubre 2020 & 825 & 3428 & 0,24 \\
\hline
\end{tabular}


Tabla VIII. 10 títulos más prestados (junio 2019-mayo 2020) por orden de reservas

\begin{tabular}{|c|c|c|c|c|c|c|c|}
\hline Título & Préstamos & Reservas & $\begin{array}{l}\text { Vista } \\
\text { Previa }\end{array}$ & Visitas & $\begin{array}{c}\text { Ratio de } \\
\text { visitas/ } \\
\text { préstamos }\end{array}$ & $\begin{array}{c}\text { Ratio de } \\
\text { reservas/ } \\
\text { licencias }\end{array}$ & Mes \\
\hline La madre de Frankenstein & 42 & 147 & 259 & 1752 & 41.71 & 1.88 & Marzo \\
\hline Un fuego azul & 45 & 103 & 127 & 717 & 15.93 & 0.51 & Marzo \\
\hline La cara norte del corazón & 35 & 80 & 18 & 758 & 25.65 & 4.0 & Diciembre \\
\hline Loba negra & 25 & 61 & 19 & 457 & 18.28 & 0.31 & Enero \\
\hline La cara norte del corazón & 16 & 61 & 15 & 744 & 46.5 & 3.05 & Octubre \\
\hline Alegría & 30 & 60 & 21 & 636 & 21.2 & 0.77 & Enero \\
\hline Terra Alta & 25 & 59 & 24 & 756 & 30.24 & 0.3 & Enero \\
\hline Si esto es una mujer & 19 & 49 & 11 & 740 & 38.94 & 0.25 & Junio \\
\hline La mansión de los chocolates & 124 & 48 & 148 & 570 & 4.59 & 0.23 & Mayo \\
\hline La chica de nieve & 131 & 46 & 129 & 215 & 1.64 & 0.24 & Abril \\
\hline
\end{tabular}

Tabla IX. 10 títulos más prestados (junio 2019-mayo 2020) por orden de ratio de reservas por licencia

\begin{tabular}{|c|c|c|c|c|c|c|c|}
\hline Título & Préstamos & Reservas & $\begin{array}{c}\text { Vista } \\
\text { Previa }\end{array}$ & Visitas & $\begin{array}{c}\text { Ratio de } \\
\text { visitas/ } \\
\text { préstamos }\end{array}$ & $\begin{array}{c}\text { Ratio de } \\
\text { reservas/ } \\
\text { licencias }\end{array}$ & Mes \\
\hline La danza de los tulipanes & 30 & 24 & 2 & 217 & 7.23 & 8.0 & Diciembre \\
\hline El latido de la tierra & 17 & 20 & 0 & 109 & 6.41 & 6.67 & Noviembre \\
\hline En el nombre del hijo & 15 & 14 & 2 & 83 & 5.53 & 4.67 & Junio \\
\hline El latido de la tierra & 18 & 13 & 1 & 80 & 4.44 & 4.33 & Octubre \\
\hline La cara norte del corazón & 35 & 80 & 18 & 758 & 21.65 & 4.0 & Diciembre \\
\hline La hija de la española & 15 & 19 & 2 & 208 & 13.86 & 3.8 & Agosto \\
\hline El último barco & 16 & 29 & 2 & 141 & 8.81 & 3.63 & Octubre \\
\hline El último barco & 17 & 25 & 2 & 183 & 10.76 & 3.13 & Noviembre \\
\hline La cara norte del corazón & 16 & 61 & 15 & 744 & 46.5 & 3.05 & Octubre \\
\hline El carrusel de las confusiones & 17 & 15 & 1 & 176 & 10.35 & 3.0 & Junio \\
\hline
\end{tabular}

más baja $(0,35)$ se produjo en febrero de 2020 y la más alta $(0,55)$ en noviembre de 2019 .

Con el confinamiento, la ratio de marzo se incrementa un $85,7 \%$ respecto a febrero y la de abril un $47,7 \%$ respecto a la del mes anterior. La tasa de abril, con casi 1 reserva por usuario activo, da cuenta de la presión de la demanda durante este periodo de confinamiento.

Los resultados de la apertura de las licencias se hacen evidentes en mayo, con una disminución del $57,3 \%$ respecto a abril. El descenso es aún más acusado en junio y julio. Agosto alcanza un nivel muy similar al de mayo, si bien en septiembre y octubre la ratio vuelve a disminuir, quizá motivado por el borrado de usuarios en septiembre.

Nuevamente, la ratio más baja de la serie es la de julio de 2020. Si la comparamos con la de julio de 2019 , el descenso es de un $-76,5 \%$.
Por último, es necesario analizar el impacto de este modelo de adquisición en los títulos más demandados mensualmente.

Si obtenemos la lista de los 10 libros más prestados cada mes desde junio de 2019 a mayo de 2020 (véase tabla VIII) y la ordenamos por número de reservas mensuales tomando los 10 más reservados, se observa un gran número de visitas, con ratios de visitas/préstamos superiores a 15 , salvo en abril y mayo. Sin embargo, el número de préstamos materializados son, en comparación con el de visitas, escasos (Tabla VIII).

Así mismo, si aplicamos la ordenación de los más prestados a partir de la ratio de reservas por licencia (véase tabla IX), se aprecia cómo las 10 primeras posiciones corresponden a los meses de junio a diciembre y el número de visitas sigue siendo muy superior al de préstamos (Tabla IX). 
Tabla X. 10 títulos más prestados (junio 2019-mayo 2020) por orden de préstamos

\begin{tabular}{|c|c|c|c|c|c|c|c|}
\hline Título & Préstamos & Reservas & $\begin{array}{l}\text { Vista } \\
\text { Previa }\end{array}$ & Visitas & $\begin{array}{c}\text { Ratio de } \\
\text { visitas/ } \\
\text { préstamos }\end{array}$ & $\begin{array}{l}\text { Ratio de } \\
\text { reservas/ } \\
\text { licencias }\end{array}$ & Mes \\
\hline La madre de Frankenstein & 185 & 38 & 145 & 244 & 1.31 & 0.49 & Abril \\
\hline A corazón abierto & 153 & 34 & 276 & 541 & 3.53 & 0.1 & Abril \\
\hline Nueve perfectos desconocidos & 150 & 0 & 95 & 326 & 2.17 & 0.0 & Mayo \\
\hline La chica de nieve & 131 & 46 & 129 & 215 & 1.64 & 0.24 & Abril \\
\hline La belleza del mal & 127 & 0 & 101 & 485 & 3.81 & 0.0 & Mayo \\
\hline Y Julia retó a los dioses & 124 & 23 & 188 & 731 & 5.89 & 0.14 & Abril \\
\hline La mansión de los chocolates & 124 & 48 & 148 & 570 & 4.59 & 0.23 & Mayo \\
\hline Un cuento perfecto & 122 & 35 & 150 & 199 & 1.63 & 0.45 & Abril \\
\hline Loba negra & 118 & 20 & 63 & 99 & 0.83 & 0.1 & Mayo \\
\hline Reina roja & 115 & 21 & 69 & 114 & 0.99 & 0.11 & Mayo \\
\hline
\end{tabular}

Sin embargo, si ordenamos los 10 títulos más prestados cada mes y tomamos los que ocupan las 10 primeras posiciones (véase tabla $X$ ), éstas están copadas por los libros prestados en abril y mayo. Así mismo, la ratio de visitas/préstamos no supera, en ningún caso, los 6 puntos.

Debemos destacar el hecho de que los préstamos de los títulos con más reservas y con una mayor ratio de reservas por licencia se produjeron, salvo en dos casos, antes de abril de 2020. Estos títulos también acumulan menos vistas previas que los libros más prestados.

Estos datos parecen indicar que cuando un libro despierta un mayor interés (más visitas) y no está disponible para su préstamo inmediato, el usuario tiende a desistir con más facilidad y no emplea la funcionalidad de vista previa.

\section{CONCLUSIONES}

La evolución del préstamo electrónico ha seguido una progresión ascendente desde la puesta en funcionamiento de estos servicios en las bibliotecas públicas españolas. No obstante, el impacto de la lectura digital entre sus usuarios ha demostrado ser bajo si lo contextualizamos dentro de la totalidad de préstamos que se producen anualmente en las bibliotecas. Así mismo, la comparación con otros países de nuestro entorno conduce a una conclusión similar.

Esta tendencia hacia un lento asentamiento de la lectura digital entre una proporción pequeña de usuarios de bibliotecas, sin embargo, podría haberse roto durante el confinamiento. El cierre de las bibliotecas públicas y de las librerías propició que el libro en formato electrónico se erigiera como una alternativa válida para un mayor número de lectores. De este modo, los servicios de préstamo digital asumieron el papel de socializadores de la lectura desempeñado por las bibliotecas públicas, tal y como muestran los datos de préstamos y de usuarios activos en marzo y abril.

Si bien a lo largo de mayo se produjo un descenso, ha sido en el mes de junio cuando ha tenido lugar el mayor decrecimiento. Son dos los elementos que pueden explican esta evolución:

- El fin del confinamiento permitió ampliar la oferta cultural y de ocio presencial fuera del hogar (reapertura de bibliotecas, cines, museos, teatros, etc.). Con ello, y a falta de estudios que analicen los hábitos de lectura durante el confinamiento, cabe esperar que se haya producido una disminución en el tiempo dedicado a esta práctica, tal y como reflejan los datos de préstamo y usuarios activos de los servicios de préstamo digital, muy especialmente en junio.

- El acuerdo con las editoriales para posibilitar el préstamo con usos concurrentes de las nuevas adquisiciones finalizó el 21/06/2020. Si bien varios editores han mantenido esta modalidad de compra, la gran mayoría ha vuelto a ofertar su catálogo en las mismas condiciones que antes de la pandemia.

El borrado de lectores que se llevó a cabo en septiembre, sin embargo, no ha tenido impacto en el número de préstamos y usuarios activos y éstos se han mantenido estables desde junio (en una cifra muy superior a los anteriores a la irrupción de la pandemia). Podemos concluir, por tanto, que el confinamiento ha dejado tras de sí un aumento significativo de usuarios activos respecto a los existentes antes del estado de alarma.

No obstante, se requiere de una perspectiva temporal más amplia que permita corroborar este impacto a 
medio y largo plazo y analizar en qué medida los elementos apuntados inicialmente como posibles condicionantes negativos para el despegue de la lectura digital (una menor oferta de títulos en formato digital, las dificultades técnicas motivadas por la atomización del acceso y la descarga de libros, y la resistencia al cambio por parte de algunos lectores y profesionales bibliotecarios) han podido quedar relegados durante el confinamiento y superados tras el mismo. Debemos apuntar, sin embargo, la dificultad de esta tarea tras la interrupción del servicio en eBiblio y las incidencias técnicas surgidas desde entonces. Todos estos problemas ponen de manifiesto la fragilidad de los servicios de préstamo electrónico, sujetos a condicionantes tales como la política de venta de las editoriales, los cambios en las empresas proveedoras de la tecnología que sustenta estas plataformas, etc. Este último aspecto requiere de un análisis en profundidad que permita valorar el impacto que puedan tener, en los datos de préstamo y usuarios activos, las dificultades vinculadas a la migración.

Así mismo, si bien la apertura de licencias ha demostrado tener un efecto positivo, es necesario valorar su mantenimiento desde el punto de vista de su sostenibilidad económica. Este análisis debe tener en cuenta el número de licencias adquiridas para reponer las que se han consumido y los costes de esta reposición, así como la viabilidad de volver únicamente a este sistema o la conveniencia de combinarlo con otras modalidades. Por tanto, la continuidad de este modelo de adquisición depende no sólo de la voluntad de los editores y distribuidores de contenidos digitales, sino también de que las administraciones sean capaces de sostenerlo económicamente. En cualquier caso, y a la luz de los resultados, el modelo de compra de licencias concurrentes limitadas vigente antes del estado de alarma difícilmente podrá satisfacer las necesidades de los usuarios activos si su número se mantiene en niveles similares a los actuales. Encontrar el equilibrio entre los recursos económicos disponibles y una adecuada respuesta a la demanda será, posiblemente, uno de los aspectos más complejos de conjugar. La vía de la flexibilidad en los modelos de venta (licencias con y sin usos concurrentes, pago por uso siempre que los precios se adecúen a los de venta al público y no tripliquen éstos como sucede actualmente) se erige como una posible solución ante los retos a los que se enfrentan las plataformas de préstamo electrónico.

Por último, esta perspectiva en el tiempo también aportará una mayor comprensión del impacto del confinamiento, así como de las medidas de restricción posteriores, en los hábitos de lectura de la población: en qué medida se ha producido una apertura hacia la lectura en formato digital o si estamos ante una respuesta meramente coyuntural.

\section{REFERENCIAS}

Alonso-Arévalo, J.; Cordón-García, J.A. (2015). Retos en torno al préstamo de libros digitales en bibliotecas. Anales de Documentación, 18(1). http://dx.doi. org/10.6018/analesdoc.18.1.207171 [Fecha de consulta: $26 / 01 / 2021$

Consellería de Cultura, Educación e Universidade (2018). Informe Galiciale. Disponible en: https://rbgalicia.xunta.gal/sites/default/files/documents/paxina/informe_galiciale_2018.pdf [Fecha de consulta: 26/01/2021]

Consellería de Cultura, Educación e Universidade (2019). Informe GaliciaLe. Disponible en: https://rbgalicia. xunta.gal/sites/default/files/documents/paxina/informe_galiciale_2019_v.2.pdf [Fecha de consulta: 26/01/2021]

Giblin, R. ; Kennedy, J.; Weatherall, K.; Gilbert, D.; Thomas, J.; Petitjean, F. (2019). Available, but not accessible? Investigating publishers' e-lending licensing practices. Information research, 24(3), paper 837. https:// doi.org/10.31228/osf.io/3u72e

Kompetenznetzwerk für Bibliotheken (2019). Die Deutsche Bibliotheksstatistik. Disponible en: https:// www.bibliotheksstatistik.de/ [Fecha de consulta: 26/01/2021]

Ministerio de Cultura y Deporte (2019a). Bibliotecas públicas españolas en cifras. Disponible en: http://www. culturaydeporte.gob.es/cultura/areas/bibliotecas/mc/ ebp/portada.html [Fecha de consulta: 26/01/2021]

Ministerio de Cultura y Deporte (2019b). eBiblio en cifras. Disponible en: http://www.culturaydeporte.gob. es/cultura/areas/bibliotecas/mc/eBiblio/en-cifras.html [Fecha de consulta: 26/01/2021]

Ministerio de Cultura y Deporte (2019c). Panorámica de la edición española de libros 2018. Análisis sectorial del libro. Disponible en: https://sede.educacion.gob. es/publiventa/descarga.action?f_codigo_agc $=16295 \mathrm{C}$ [Fecha de consulta: 26/01/2021]

Nasjonalbiblioteket (2019). Statistikk for folkebibliotek. Disponible en: https://kunnskapsbase.bibliotekutvikling.no/statistikk/ [Fecha de consulta: 26/01/2021]

Netbib (2019). Statistik for eReolen Disponible en: https://netbib.dk/statistik-ereolen [Fecha de consulta: 26/01/2021]

Opetus-ja kulttuuriministeriö (2019). Suomen yleisten kirjastojen tilastot. Disponible en: http://tilastot.kirjastot.fi/?lang=fi [Fecha de consulta: 26/01/2021]

Revelle, A.; Messner, K; Shrimplin, A.; Hurst, S. (2012). Book lovers, technophiles, pragmatists, and printers: The social and demographic structure of user attitudes toward e-books". College \& Research Libraries, 73(5), 420-429.

Sánchez-Muñoz, E. (2018). Acceso a las plataformas de préstamo digital: integración vs. atomización. El Profesional de la Información, 27(3), 582-594. https://doi. org/10.3145/epi.2018.may.11

Sánchez-Muñoz, E. (2019). De la proto-lectura a la lectura digital: transfiguraciones y mutaciones. En J.A. Cordón-García (coord.). Libro, lectores y lectura digital, 155-180. Madrid: Instituto Juan Andrés de Comparatística y Globalización; Casimiro Libros 
Sánchez-Muñoz, E. (2020). La biblioteca pública como dinamizadora de la lectura digital. En: Díez Mediavilla, A.; y Gutiérrez Fresneda, R. (coords.). Lectura y dificultades lectoras en el siglo XXI. Barcelona: Octaedro. ISBN: 978-84-18348-54-9

Sveriges Nationabibliotek (2019). Biblioteksstatistik. Disponible en: https://www.kb.se/ samverkan-och-utveckling/biblioteksstatistik/ hamta-ut-statistik/statistikrapporter.html\#i- tem-42705879d169b8ba882a1c9a [Fecha de consulta: $26 / 01 / 2021]$

Valbuena, J.; Cordón-García, J.A. (2019). El impacto de los contenidos digitales en los espacios físicos: el proyecto Nubeteca y la recuperación de la biblioteca como lugar de encuentro. Periférica: Revista para el análisis de la cultura y el territorio, 20, 302-311. Disponible en: https://revistas.uca.es/index.php/periferica/article/ view/5599/5879 [Fecha de consulta: 26/01/2021] 